\title{
Fractal parameters and SAR images
}

\author{
Gerardo Di Martino, Graduate Student Member, IEEE, Antonio Iodice, Senior Member, IEEE, \\ Daniele Riccio, Senior Member, IEEE and Giuseppe Ruello, Member, IEEE \\ Dipartimento di Ingegneria Elettronica e delle Telecomunicazioni, Università di Napoli "Federico II" \\ Via Claudio 21, 80125, Napoli, Italy \\ E-mail: \{gerardo.dimartino, iodice, daniele.riccio, ruello\}@unina.it
}

\begin{abstract}
The fractal geometry proved to be the most appropriate mathematical instrument in describing natural scenes, by means of few effective and reliable geophysical parameters. In this paper we describe a complete processing chain for the retrieving of SAR image fractal parameters and for change detection purposes. We present an overall framework employing fractal based models, algorithms and tools to support identification of natural area changes due to natural or manmade disasters. In addition, we test our fractal framework in a simulated disaster scenario. In particular, we consider the case of a simulated volcano eruption scenario to test the potentialities of our technique for lava flow detection. We also compare the performances of the proposed framework on different kinds of disasters to stress the significant differences in the parameters used for change detection purposes.
\end{abstract}

Index Terms-Synthetic Aperture Radar, Fractals, Disaster Monitoring

\section{INTRODUCTION}

In recent years the interest in the application of remote sensing techniques for the monitoring and assessment of natural disasters has been growing very fast. This is due to the fact that remote sensing instruments provide a huge amount of data relevant to almost any area of the Earth.

In particular, SAR sensors are very well suited for the observation of this type of phenomena, because the microwave frequencies employed ensure all-weather, all-time capabilities. Furthermore the scales involved in the formation of the SAR image are the same affected by dramatic changes in case of disaster. However, the difficulty in interpretation of these data still limits their use to expert observers.

Several approaches for data interpretation are presented in literature [1]. Most of them are based on empirical analyses of remote sensing data, essentially driven by user needs. These analyses are generally supervised and, to be effective, should be performed only by people with a remarkable level of competence both on the sensors and on the effects of different disasters on the environment. Indeed, when a disaster occurs it dramatically modifies the surface profile of the observed scene from scales comparable to the sensor resolution up to scales comparable to the electromagnetic wavelength. Besides of these geometrical changes, also the electromagnetic characteristics of the scene abruptly change: consequently remote sensing data are affected by textural modifications and, at least in principle, it should be possible to detect these changes on the images.
In order to develop tools for the extraction of value added information from remotely sensed images, a better insight into the mechanisms and parameters governing SAR image formation is necessary. In order to improve the understanding of SAR images and to define the parameters of interest in case of disaster it is of key importance to introduce appropriate models for the description both of the imaged surface and of the signal backscattered from it. Only in this way we can try to relate significant parameters of the observed scene to textural characteristics in the SAR image, thus preparing the way to new detection techniques no more based simply on a morphological analysis but on the definition of relevant "physical" parameters on the image.

The fractal geometry [2] proved to be the most appropriate mathematical instrument to quantitatively describe natural surfaces. It simply accounts for the complexity of the scene, by means of few effective and reliable geophysical parameters. In this paper we assume a fractal model for the imaged surface and we compute analytically the scattering from this kind of surfaces as stated in [3]. The key concepts of these models are presented in the next Section. All the above mentioned models are part of the SAR raw-signal simulator developed and tested at the University of Naples, Italy [4].

As for the inverse models, in this paper we describe a complete processing chain for the retrieving of SAR image fractal parameters and for change detection purposes. Our inverse chain allows the estimation of several fractal characteristics of SAR images. From one side, we can retrieve the fractal parameters of the image and then compare between pre- and post-crisis SAR image fractal parameters. In this way we can develop and test several fractal-based change detection techniques: as stated above, this approach presents a major advantage with respect to the classical ones, i.e. the considered parameters hold a physical meaning and could be related to fractal parameters variations on the imaged scene. Furthermore, in particular cases, we can evaluate the fractal dimension of the contour of a region of interest (ROI), for example in case of zones identified as hit by lava flows, it is possible to discriminate between different kinds of lava analyzing the fractal dimension of the contour.

Finally, in the last Section of the paper, we test our fractal framework in a simulated disaster scenario. In particular, we consider the case of a simulated volcano eruption scenario to test the potentialities of our technique for lava flow detection. We also compare the performances of the proposed framework on different kinds of disasters; in particular, we consider the 
identification of a flooded zone [5], to stress the significant differences in the parameters used for change detection purposes.

\section{DIRECT MODELS}

In this Section we present the fractal framework and we outline the rationale of the proposed method.

\section{A. SAR simulation}

In past years, a SAR raw signal simulator was developed and tested [4], [6]. A SAR data simulator is an important tool to help scientists in better understanding the mechanisms underlying SAR image formation and in the interpretation of SAR data textures and of their modifications.

In the following we describe briefly the key issues for SAR signal simulation. Let $x$ and $r$ be the independent space variables, standing respectively for azimuth and range. By using primed coordinates for the independent variables of the SAR raw signal, $s\left(x^{\prime}, r^{\prime}\right)$, this can be expressed as [3]:

$$
s\left(x^{\prime}, r^{\prime}\right)=\iint d x d r \gamma(x, r) g\left(x^{\prime}-x, r^{\prime}-r ; r\right),
$$

where $\gamma(x, r)$ is the reflectivity pattern of the scene and $g\left(x^{\prime}-x, r^{\prime}-r ; r^{\prime}\right)$ the unit impulse response of the SAR system [4], [6]. Evaluation of the reflectivity pattern requires a description of the observed surface as well as a model for their interaction with the electromagnetic fields radiated by the SAR antenna [4]. In this paper we use the models presented in the next two paragraphs.

\section{B. Fractal surface model}

Fractal models are widely considered the most appropriate to quantitatively describe natural surfaces [2]. Fractal geometry is able to reliably account for the non-stationarity of natural surfaces, as well as for their self-affinity. The most used fractal model is the fractional Brownian motion $(\mathrm{fBm})$ [3]. The $\mathrm{fBm}$ is defined in terms of the probability density function of its height increments: a stochastic process $z(x, y)$ is an $\mathrm{fBm}$ surface if, for every $x, y, x^{\prime}, y^{\prime}$, it satisfies the following relation:

$$
\operatorname{Pr}\left\{z(x, y)-z\left(x^{\prime}, y^{\prime}\right)<\bar{\zeta}\right\}=\frac{1}{\sqrt{2 \pi} s \tau^{\prime \prime}} \int_{-\infty}^{\bar{\zeta}} \exp \left(-\frac{\zeta^{2}}{2 s^{2} \tau^{2 \prime \prime}}\right) d \zeta
$$

where $\tau$ is the distance between the points $(x, y)$ and $\left(x^{\prime}, v^{\prime}\right)$, and the two parameters that control the $\mathrm{fBm}$ behaviour are:

$H$ : the Hurst coefficient $(0<H<1)$, related to the fractal dimension $D$ by means of the relation $D=3-H$.

$s:$ the standard deviation, measured in $\left[\mathrm{m}^{(1-H)}\right]$, of surface increments at unitary distance, a real parameter related to an $\mathrm{fBm}$ characteristic length, the topothesy $T$, by means of the relation $s=T^{(l-H)}$.
To compute the reflectivity function of the observed scene the considered simulator requires as input a DEM relative to the scene of interest, sampled with a resolution coherent with the considered sensor parameters: in practice, we need to interpolate the available DEM [6].

We use the $\mathrm{ABm}$ to model the surface imaged by the SAR sensor. Hence, the fractal parameters, retrieved from the considered DEM, are used to perform a stochastic fractal interpolation on the available DEM. In this way, the interpolated DEM inherits the fractal behaviour of the original surface [6]. The use of this particular interpolation technique provides the possibility to use, for example, the interferometric DEMs from SRTM (freely downloadable on the internet) or any low resolution DEM, if a higher resolution one is not available.

\section{Fractal scattering model}

Theoretical [2] and experimental [8] studies suggest that use of fractal models improve the scattering method results. In this paper we use the $\mathrm{fBm}$ fractal model in describing the surface roughness and the small perturbation method (SPM) as scattering model for evaluating the reflectivity pattern [3].

Comparison between simulated and actual SAR data was presented in [6] with respect to image single point normalised moments and autocorrelation function, thus assessing the simulator reliability. In those comparisons the fractal parameters accounting for the microscopic description of the scene were assumed to be constant. In this case the extension we propose allows considering for the microscopic scale (up to the electromagnetic wavelength scale) the fractal parameters estimated from the available DEM, so that in order to compute the reflectivity function we use fractal parameters varying all over the scene of interest [5].

\section{Comparison with actual SAR images}

In order to show the ability of the presented simulator in reproducing SAR data, we show some meaningful simulation results. In particular, for one of the simulations the actual SAR image relevant to the simulated scenario is available. The region of interest is the area of Maratea $\left(39^{\circ} 59^{\prime} \mathrm{N} 15^{\circ} 42^{\prime} \mathrm{E}\right.$ ), South of Italy, a coastal area surrounded by steep mountains. A digital elevation model (DEM) of a $20 \times 20 \mathrm{Km}^{2}$ area, with 20 x $20 \mathrm{~m}^{2}$ pixel spacing was available for the considered area, as well as an ERS-1 C-band SAR image, acquired in descending orbit on the 30 of January 1996 , with a view angle of $24.88^{\circ}$. The DEM was interpolated via the fractal approach introduced in [5], in order to be described on a rectangular grid, with pixel dimensions of $3.99 \times 19.9 \mathrm{~m}^{2}$, in accordance with the ERS-1 acquisition geometry. The microscopic roughness, responsible for the electromagnetic scattering, is provided by estimating the local fractal dimension of the DEM. Therefore, we simulated the SAR image acquired by the ERS-1 sensor. The dielectric constant $\varepsilon$ of the scene is set to $\varepsilon=4 \varepsilon$, and the conductivity $\sigma$ is set to $\sigma=0.001 \mathrm{~S} / \mathrm{m}$.

The image obtained from the simulated raw signal via standard processing, is presented in Fig. 1 and compared with the actual image provided by the ERS-1 SAR of Fig. 2 (near range is on the left). The images are averaged with a $2 \times 10$ 
multi-look, so that the presented image resolution in azimuth slant range is $39.86 \times 15.81 \mathrm{~m}^{2}$, which corresponds to an azimuth - ground range almost square pixel $\left(39.86 \times 37.58 \mathrm{~m}^{2}\right)$. A visual comparison shows the capacity of the simulator of reproducing the main characteristics of the SAR image, suggesting the use of the SARAS as support for SAR actual image interpretation.

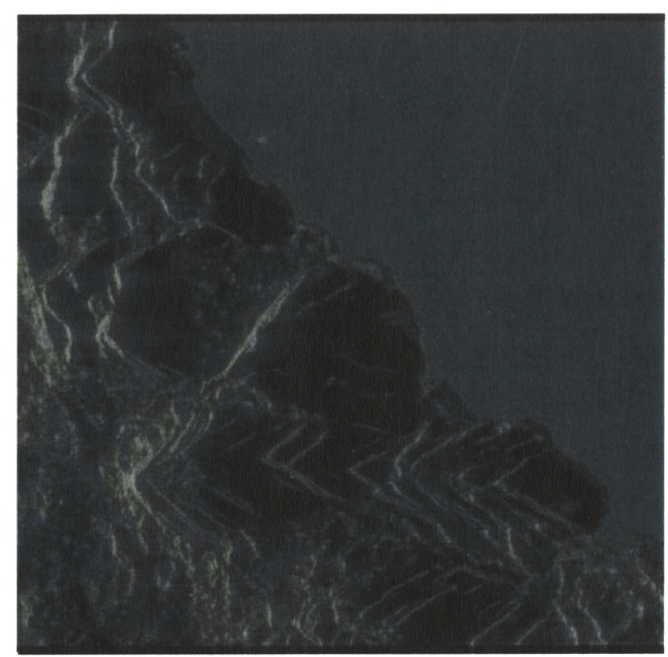

Figure 1. Simulated SAR image of the region of interest.

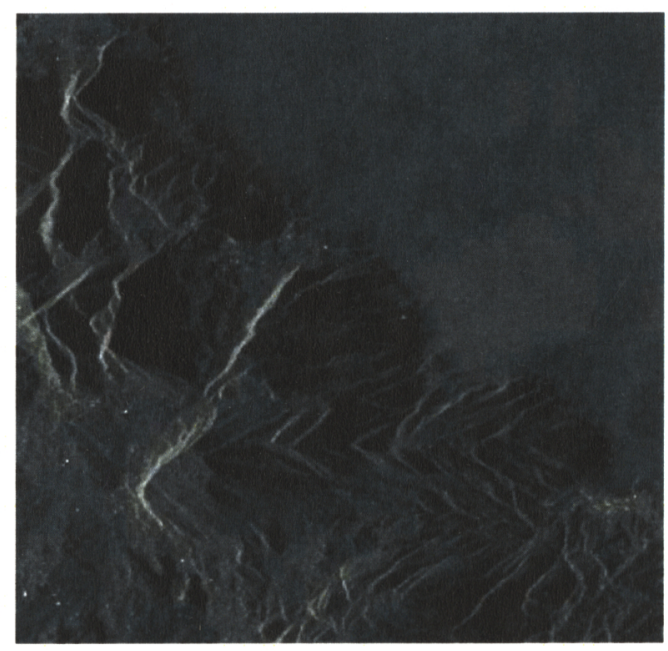

Figure 2. Actual SAR image of the region of interest.

\section{INVERSE MODELS}

In the open literature, several methods for the extraction of information from SAR images relative to scenarios subject to changes are proposed. The majority of them are based on classical magnitude change detection techniques such as ratioing and differencing [1].

In this paper we present a technique for the extraction of fractal parameters, whose use can bring value added information. In particular, we propose a twofold extraction of fractal parameters, one based on the intensity variation, the other on the extraction of the fractal parameters of a ROI contour.

\section{A. SAR image fractal dimension extraction}

An analytical relationship between the fractal dimension of a portion of surface and the fractal dimension of the corresponding SAR image has not yet been deeply investigated. Anyway, the use of the SAR simulator allows the definition of a wide set of canonical scenes, which can provide an empirical relationship [5].

To carry on these studies, an efficient estimation technique is needed. Our algorithm is based on the variogram analysis. For a given surface, the structure function (variogram) $V(\tau)$ is defined as the mean square increment of elevation points placed at distance $\tau$ :

$$
V(\tau)=\left\langle\left(z(x, y)-z\left(x^{\prime}, y^{\prime}\right)\right)^{2}\right\rangle,
$$

The variogram of an $\mathrm{fBm}$ surface can be evaluated in terms of the parameters $H$ and $s$ [3] and expressed in logarithmic form as:

$$
\log V(\tau)=2 \log s+2 H \log \tau,
$$

which defines in a log-log plane a linear behaviour with slope $2 H$, and ordinate intercept $2 \log s$. Such a dependence leads to retrieve the fractal parameters with a linear regression over the log-log plots of measured values of $V(\tau)$ [3].

Note that the application of this technique to SAR images is someway critical, because it is necessary to take into account the non equal spacing of the data set. We enlarged the available technique validity to deal with such a case.

\section{B. Extraction of the ROI contour fractal dimension}

In several applications, different regions of interest can be classified in accordance with the roughness of their contour. To this aim, a box counting algorithm can be employed, provided that the region of interest was previously defined.

This operation can be not straightforward on SAR images due to the presence of speckle. Therefore, a pre-processing step, possibly based on the techniques presented in the previous section, is required. We exploited here the simulator ability of generating a training set for understanding the technique performances and limitation. As an example, we generated two areas of different contour fractal dimension, as presented in Fig. 3. The left and the right areas have a contour fractal dimensions of $D=1.27$, and $D=1.3$, respectively.
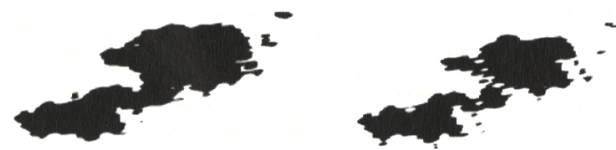

Figure 3. Simulated areas with fractal dimension $D=1.27$ (on the left) and $D=1.3$ (on the right). 
These masks can be used to define areas with different geometrical (fractal dimension and topothesy) and dielectric (permittivity and conductivity) parameters with respect to a homogeneous background. At this point, several techniques can be used to extract the contour of the ROI. In particular, the variogram technique used in the estimation of the fractal dimension presents edge detection capabilities that can be used to this aim. Once the corresponding area is retrieved in the SAR image, we can estimate its contour fractal dimension. For the proposed example, we obtained $D_{s}=1.23$ and $D_{s}=1.27$, respectively. As expected the fractal dimension is almost the same. An underestimation is mainly due to the filtering of the speckle necessary to extract the area from the SAR image.

\section{THE CASE Studies}

In the following, the potentiality of the chain presented in the previous section is tested on a case study, focused on the monitoring of volcanic areas. In particular, a novel approach is proposed, based on change detection techniques applied to fractal parameters retrieved from pre- and post-crisis images. In the last part of the Section, a comparison of the technique performances in case of a different kind of disaster, in particular the identification of a flooded zone, is presented.

\section{A. Volcano eruptions}

In the following, we present two simulated volcano eruption scenarios. For both cases we show the performance of our fractal approach for the identification of areas covered by the lava flows.

The first case is relevant to the Kilimanjaro volcano $\left(2^{\circ} \mathrm{S}\right.$ $36^{\circ} \mathrm{E}$ ). We provided as input for the simulator the digital elevation model (DEM) of the zone, on a $90 \times 90 \mathrm{~m}^{2}$ grid. The DEM was interpolated via the fractal approach, in order to be in accordance with the ERS-1 acquisition geometry. In Fig. 4 we present a $3 \mathrm{D}$ representation of the DEM.

The simulation was performed according to the procedure presented in the previous Section. The simulated image is shown in Fig. 5 (near range is on the left). Such an image represents a reference for the situation, in absence of lava flows.

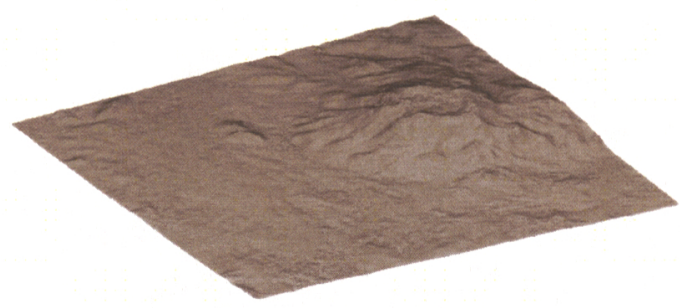

Figure 4. 3D representation of the Kilimanjaro volcano area.

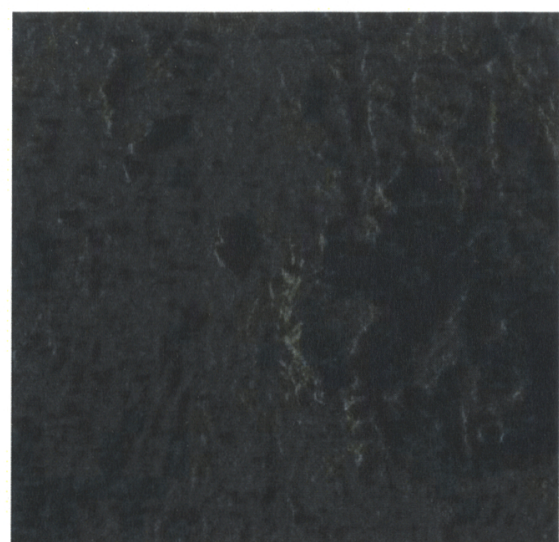

Figure 5. Simulated ERS-1 image of the Kilimanjaro area

In order to estimate the ability of the proposed technique, we simulated a lava eruption, by setting the simulation parameters of a given region to the typical values for the most common lava flows (AA and pahoehoe). However, to appropriately account for the presence of lava, we gave the region parameters the values defined in Table 1.

TABLE I. LAVA PARAMETERS
\begin{tabular}{|l|c|c|}
\hline \multicolumn{1}{|c|}{ Lava parameters } & $\boldsymbol{A A}$ & Pahoehoe \\
\hline Diclectric Constant & 8 & 20 \\
\hline Conductivity [S/m] & 0.01 & 1 \\
\hline Hurst cocfficient & 0.6 & 0.85 \\
\hline $\left.\mathrm{s}\left[\mathrm{m}^{\wedge}(1-H)\right)\right]$ & 0.1 & 0.2 \\
\hline
\end{tabular}

Two kinds of lava are defined: a smoother one, recalling the properties of the pahoehoe lava flows and a rougher one, recalling the properties of the AA lava flows. In Fig. 6 and 7 the two simulated SAR images are presented (near range is on the left).

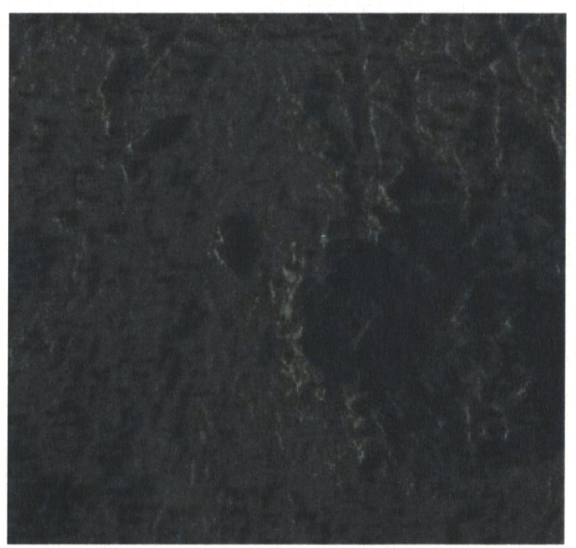

Figure 6. Simulated ERS-1 images of the Kilimanjaro area covered with pahochoc lava. 


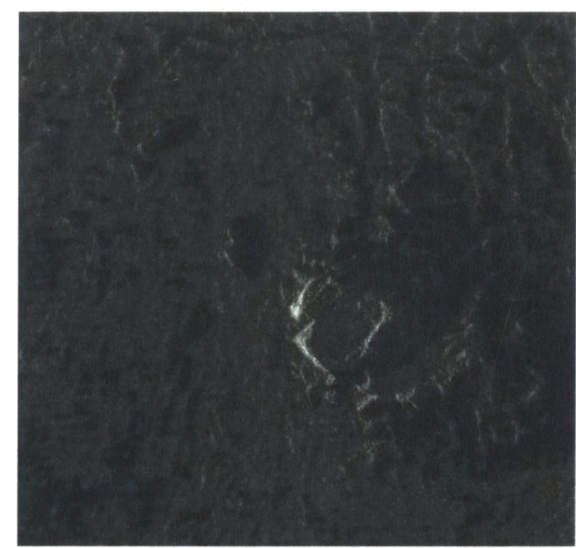

Figure 7. Simulated ERS-1 images of the Kilimanjaro area covered with pahochoc lava.

On the obtained SAR images we applied the variogram method, obtaining the maps of the fractal parameters of the two scenarios for change detection purposes. In this case, only one of the two independent fractal parameters of the image, i.e. the $s$ parameter, was used to identify the region covered by the lava. This is due to the fact that in our simulation we considered that the high scale profile is not changed by the lava flow. Anyway, the presence of the lava in the scene significantly changes the microwave scales surface characteristics of the area affected by the eruption, and it is possible to exploit the sensitivity of the SAR return to the surface microscopic roughness. We simulated the volcano eruption by changing the microscopic parameters of the surface and this resulted, essentially, in a change of intensity of the image in the zone of interest. Anyway, we will make some more observations on this issue when presenting our results concerning the case of flooding.

In order to quantify the performance of the technique, a reference map can be generated, by simulating the SAR data in absence of speckle in the pre- and post- eruption cases. Their difference defines the "ground truth" for the case study. In Fig. 8 the ground truth and the map obtained with the proposed approach are shown.

We applied our technique also to the case of Mount Cameroon volcano $\left(4^{\circ} 2^{\prime} \mathrm{N} 9^{\circ} 17^{\prime} \mathrm{E}\right)$, obtaining similar results, as shown in Fig. 9.
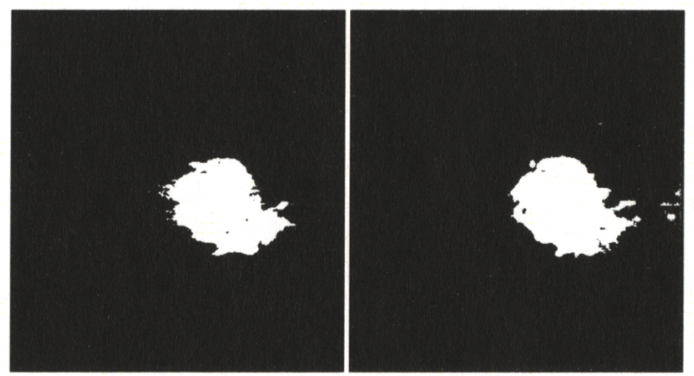

Figure 8. Comparison between ground truth map (on the left) and the classification obtained with the proposed technique (on the right) for the case of the Kilimanjaro volcano arca.
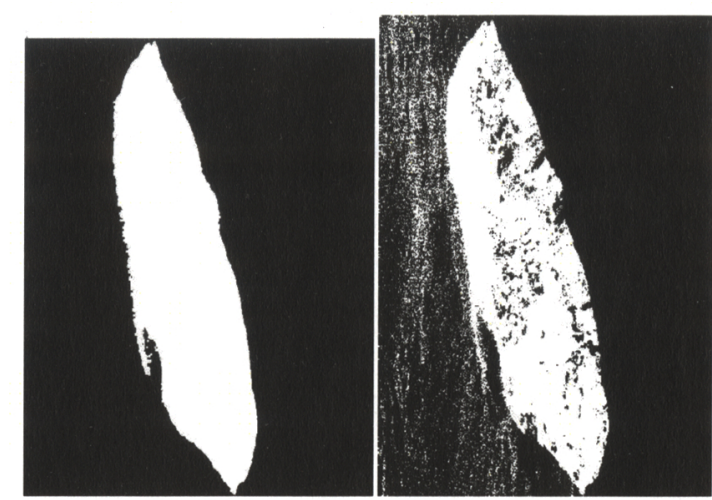

Figure 9. Comparison between ground truth map (on the left) and the classification obtained with the proposed technique (on the right) for the casc of Mount Camcroon volcano arca.

Note that in this case the false alarm rate increased with respect to the previous example. This is mainly due to a more problematic ROI, subject to severe geometrical distortion problems. Anyway, the majority of false alarm points can easily be eliminated with simple post-processing algorithms, due to their specific characteristics.

\section{B. Flooding}

As we already stated, in the previous examples only the microscopic roughness of the ROI was changed by the presence of lava. No modification to the high scale profile was supposed and this implied only a variation in the intensity of the signal backscattered from the zone hit by the disaster. This resulted in the fact that one of the fractal parameters retrieved from the image was not necessary for change detection purposes.

To better stress this issue we now present another simulated disaster scenario, relevant to a flooding event [5]. The region of interest is the one of Maratea, as presented in the previous Section. In this case we modified the original DEM by creating a river's spate in the valley shown in Fig. 10. The mean difference between the pre- and post-crisis DEM in that area is about $30 \mathrm{~m}$.

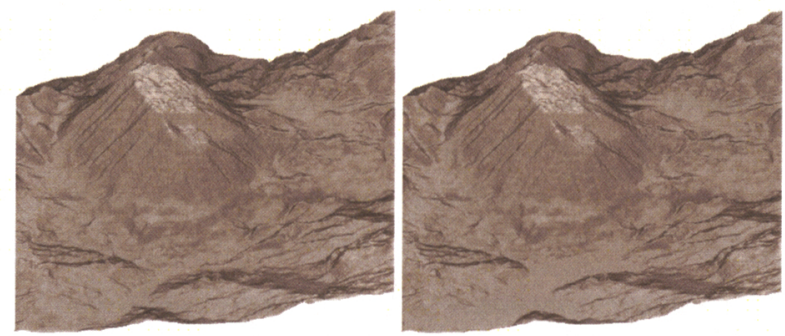

Figure 10. Close up of the area hit by the flooding.

In order to appropriately simulate the presence of water in the flooded region, we modified the microscopic roughness and the dielectric parameters as well. As far as the microscopic fractal parameters are concerned, in the areas affected by the flooding we set $H$ to a typical value for the water surface $(H=0.75)$, and we set $s$ to one half of the value in the pre-crisis 
scenario. As for the dielectric characterization, the area affected by the flooding is assumed to have a dielectric constant of $20 \mathcal{E}$, and a conductivity of $1 \mathrm{~S} / \mathrm{m}$, which are typical values for extremely wet terrain; the surrounding area is assumed to have a dielectric constant of $4 \mathcal{E}_{i}$ and a conductivity of $10^{-3} \mathrm{~S} / \mathrm{m}$, typical of terrains with low water content.

In [5] we studied this scenario using ERS VV polarization. Here we exploit the potentialities of the simulator: in fact, it allows a polarimetric analysis of the proposed technique. As an example we simulated the scenario of interest with $\mathrm{HH}$ polarization obtaining the result presented in Fig. 11 (the flooded region can be noticed at the bottom of the image, in the left corner). Anyway, in this case, the obtained image is quite similar to the one obtained with VV polarization and, consequently, we do not expect significant variations in the performance of the technique.

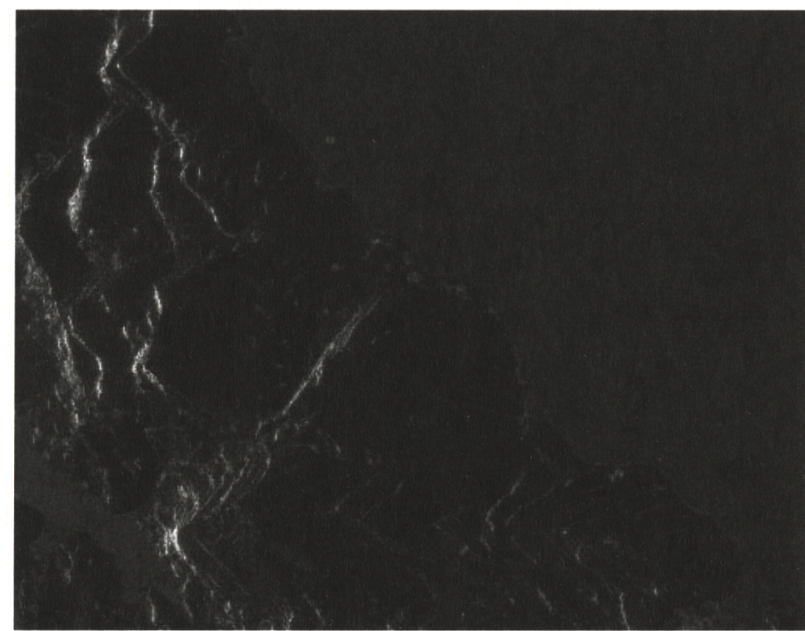

Figure 11. Simulated post-flooding image for the case the Maratea area (near range is on the left)

Finally, we applied the fractal framework obtaining the classification result presented in Fig. 12.
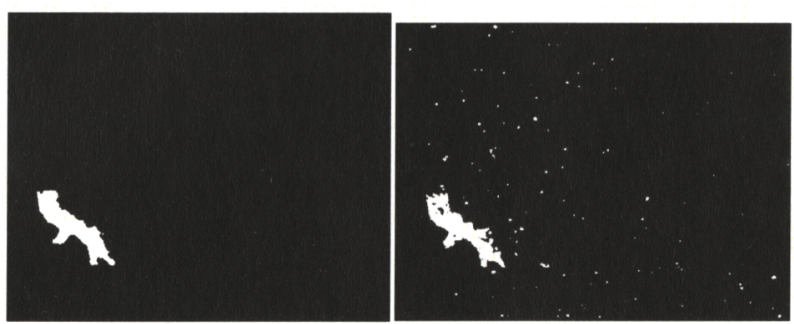

Figure 12. Comparison between ground truth map (on the left) and the classification obtained with the proposed technique (on the right) for the case of flooding in the Maratea arca.
In this case we exploited for the classification all the available parameters: a combination of the fractal parameters of the image was used to generate the binary masks presented above. The modification of the DEM at macroscopic scales determined a variation in the fractal dimension of the image and this information was complementary with respect to that supplied by the $s$ parameter [5]. Essentially the fractal dimension of the image shows a dependence on the fractal dimension of the imaged surface measured at the scales of SAR resolution rather than at wavelength scales.

\section{CONCLUSIONS}

In this paper we presented a complete framework of fractal models whose use allows improving the comprehension, interpretation, and information extraction from SAR images. In particular we addressed three case studies, showing the capability of the proposed technique to face different disaster management problems. In particular, we simulated two volcanic eruption scenarios and a flooding stressing the importance of the proposed simulation procedure to help in the understanding of SAR data formation mechanisms and to improve the parameter retrieving algorithms. We also presented a novel change detection technique based on the fractal parameters estimated on the image, stressing the difference between the proposed case studies. The obtained results show that the proposed techniques lead to significant performances in the extraction of information from SAR data.

\section{REFERENCES}

[1] E. Rignot and J. van Zyl, "Change Detection Techniques for ERS-1 SAR Data", IEEE Trans. Geosci. Remote Sens., vol. 31, no. 4, pp.896906, July 1993

[2] B.B. Mandelbrot, The Fractal Geometry of Nature, W.H.Frecman \& C., New York, USA, 1983.

[3] G. Franceschetti, A. Iodice, D. Riccio, "Fractal Models for Scattcring from Natural Surfaces". Invited Chapter in Scattering, R. Pike, P. Sabaticr, cds.. Academic Press, London (UK), pp. 467-485. Scpt. 2001.

[4] G. Franceschetti, M. Migliaccio, D. Riccio, G. Schirinzi. "SARAS: a SAR Raw Signal Simulator", IEEE Trans. Geosci. Remote Sens., vol. 30, no. 1, pp. 110-123, Jan.1992.

[5] G. Di Martino, A. Iodice, D. Riccio and G. Rucllo, "A Novel Approach for Disaster Monitoring: Fractal Models and Tools", IEEE Trans. Geosci. Remote Sens., vol. 45, no. 6, pp. 1559-1570, Junc 2007.

[6] G. Franceschetti, M. Migliaccio, D. Riccio, "SAR Raw Signal Simulation of Actual Scenes Described in Terms of Sparse Input Data", IEEE Trans. Geosci. Remote Sens., vol. 32, no. 6, pp. 1160-1169, Nov. 1994.

[7] G. Rucllo, P. Blanco, A. Iodicc, J. J. Mallorqui, D. Riccio, A. Broquetas, G. Franceschetti, "Synthesis, Construction And Validation Of A Fractal Surface" IEEE Trans. Geosci. Remote Sens., vol. 44, no. 6, pp. 14031412, Junc 2006 